${ }^{\circ}$ Entomologica Fennica. 27 August 1998

\title{
Trichoptera in Finnish biogeographical provinces
}

\author{
Erkki M. Laasonen, Leena Laasonen \& Ola Nybom
}

Laasonen, E. M., Laasonen, L. \& Nybom, O. 1997: Trichoptera in Finnish biogeographical provinces. - Entomol. Fennica 9: 115-135.

The sum of Trichoptera found in Finland is 208 species, after we here report both Crunoecia irrorata (Curtis, 1834) and Apatania stylata Navas, 1916 as new for the country. Out of the 21 biogeographical provinces there is now altogether 2520 finds, an increase of $+40.1 \%$ since the earlier paper dealing with the subject (Nybom 1960). Putting into a graph the latitude of the weighted geographical centre of a biogeographical province, and the sum of Trichopteran species found in it, and calculating a regression line, it comes up that in the north of Finland about 50 species less are found than in the south. In addition, when comparing the actual sums with this regression line, the three provinces with most incomplete investigation work are $\mathrm{Al}=$ Aland Islands, Oba = northern Ostrobothnia, southern part, and $\mathrm{Ka}=$ southern Karelia, in which the sum of finds are $-32 \%,-21 \%$, and $-19 \%$ below the regression line, respectively. Recently, the only determination key to Finnish caddisflies built up by the late J. Kyrki, Oulu University, was published in a book for fly fishers (Rinne et al. 1995).

Erkki M. Laasonen, Medical School, University of Tampere and Department of Radiology, Tampere University Hospital, POB 607, FIN-33101 Tampere, Finland

Leena Laasonen, Department of Radiology, Surgical Hospital, Helsinki University Hospital, POB 385, FIN-00029 HYKS, Finland

Ola Nybom, Kyrksundvägen 3 F 91, FIN-00840 Helsingfors, Finland

Received 19 February 1997, accepted 18 November 1997

\section{Introduction}

Significant progress in books to determine Trichoptera (Macan \& Worthington 1973, Malicky 1983, Katsalova 1987, Rinne et al. 1995) and good lists of Finnish Trichoptera (Nybom 1960, Hiilivirta \& Laasonen 1984) have initiated promising research on Finnish caddisflies. In this paper we list the old and new observations of Trichoptera in Finnish biogeographical provinces; try to find out the provinces in which a lot of work should still be done; and wish to activate entomologists, perhaps preferably amateur lepidopterologists, to take up with this group, too.

\section{Method}

We started with checking the literature on Finnish caddisflies since 1960 ( Nybom 1960). The most weighting paper presented a new species: Oxyethira klingstedtii Nybom 1983. Many papers dealt with additions or deletions to Finnish fauna (Svensson \& Tjeder 1975, Hulden et al. 1977, Ny- 
bom 1980, Silfverberg 1981 and 1986, Hiilivirta 1982, Leivo 1996) giving a sum of 206 species. New finds from these papers, as well as from those dealing with new provincial finds, were included first.

Thereafter unpublished manuscripts from all Finnish Universities were gathered. In addition, the library of the Finnish Environmental Centre in Helsinki, as well as the 13 regional environmental libraries, were checked for their reports dealing with macro-zoobenthos or bottom fauna of water bodies. As an experiment, all the 192 reports dealing with bottom fauna from Uusimaa Regional Environmental Center were went through in detail. Trichoptera were mentioned in 47 reports, but only in nine of them the analysis went up to species level. Even then, only few species like Cyrnus flavidus, Molannodes tinctus, and Oligotricha striata (reported as Phryganea striata) were notoriously repeated. Three new finds for Uusimaa (N) were found (Mettinen $1994 \mathrm{a}, \mathrm{b}$ ).

After that public as well as private collections were checked.

Finally an inquiry was sent to 23 persons, who during last 40 years had shown written or verbal activity on Finnish caddisflies. We are very happy to inform, that all of them could find time to answer to our inquiry. Here, as well as in earlier steps, the source which was printed or came to our knowledge first, was priorized.

The determination of reported specimens was checked only exceptionally. Our lists follow Andersen \& Wiberg-Larsen (1987), and Wallace (1991), with no new taxonomic analyses. However, the systematic order of tribes, genera and species by Hiilivirta \& Laasonen (1984) were retained, instead of the alphabetic order in the other checklists.

\section{Results}

The full data is presented in the Table 1 at the end of this paper. In addition, unpublished finds from manuscripts, collections and letters are listed in the Appendix. The major unpublished sources were Collection of Helsinki University with 152 new provincial finds, Coll. (and archives of) $\mathrm{Ny}$ bom 105 finds, Coll. of Oulu University 88 finds, Coll. Laasonen with 32 finds, letter from M. Hir- venoja 28 finds, and Coll. of Kuopio Museum 20 finds, respectively.

We report two species new to Finland: $\mathrm{Cru}$ noecia irrorata (Curtis, 1834) six specimens from St: Jämijärvi Uhrilähde 10.8.1996 E. Saarela leg. and det. (93) and Apatania stylata Navas, 1916, 12 specimens from Ta: Valkeakoski K. Järventausta leg. and O. Nybom det. (88). Thus the sum of Trichoptera found in Finland is now 208 species. That means that since 1960 every fifth year a new species has been found. Athripsodes bilineatus was reported twice in manuscripts dealing with pollutions in water bodies, on the basis of a larval find $(64,67)$. However, the determination of Athripsodes larvae seems to be very difficult and after discussions with the observers both these finds were omitted.

After omission of the finds from $\mathrm{Ka}$ and $\mathrm{Kl}$, which were from an area no more belonging to Finland, the net sum of provincial reports by Nybom is 1799 finds (Nybom 1960). To that we now add 721 finds, i.e. $+40.1 \%$. Out of them, 215 have already been published elsewhere. However, in the papers dealing with environmental surveys of water bodies the new provincional aspect was never mentioned - the finds had to be picked up one by one. We found 506 unpublished or unnoted finds and list them up in the Appendix. Out of the new finds, 166 were in a way technical, i.e. as a result of the split of biogeographical province $\mathrm{Ob}$ to $\mathrm{Oba}$ and $\mathrm{Obb}$, and province Lkem to Lkoc and Lkor; as well as splitting Potamophylax "stellatus" into P. latipennis and P. cingulatus.

There seems to be much work undone. Looking at the "minuses-in-between" (see Table 1), i.e the provinces in which a species has not been found, although it is found at least in two neighbouring provinces, we sum up 641 potential finds ( $25.4 \%$ out of 2520 ), which just await observation. The estimate could be even higher, as the knowledge of e.g. Hydroptilidae seems to be far from perfect. In addition, in the Table 1 there are nine species in which the new find, "the number", leaps more than $200 \mathrm{~km}$, as the crow flies, from the earlier find. However, six of these: Agraylea cognatella, Oxyethira sagittifera, O. mirabilis, Apatania zonella, Limnephilus ignavus, and Asynarchus contumax have clusters of new finds more or less far away; as well two of them Oxyethira falcata and Apatania dalecarlica are difficult to 
Figure 1: The weighted northern latitude of the Finnish biogeographical provinces on the horizontal axis and the sum of Trichoptera species found in them on the vertical axis. The regression line drawn has a correlation coefficient of $r=-0.824$. The provinces with best amount of finds are marked with an inverted triangle $(\nabla)$ and with bold. The underachieving provinces are marked with a triangle $(\mathbf{\Delta})$ and with italics.

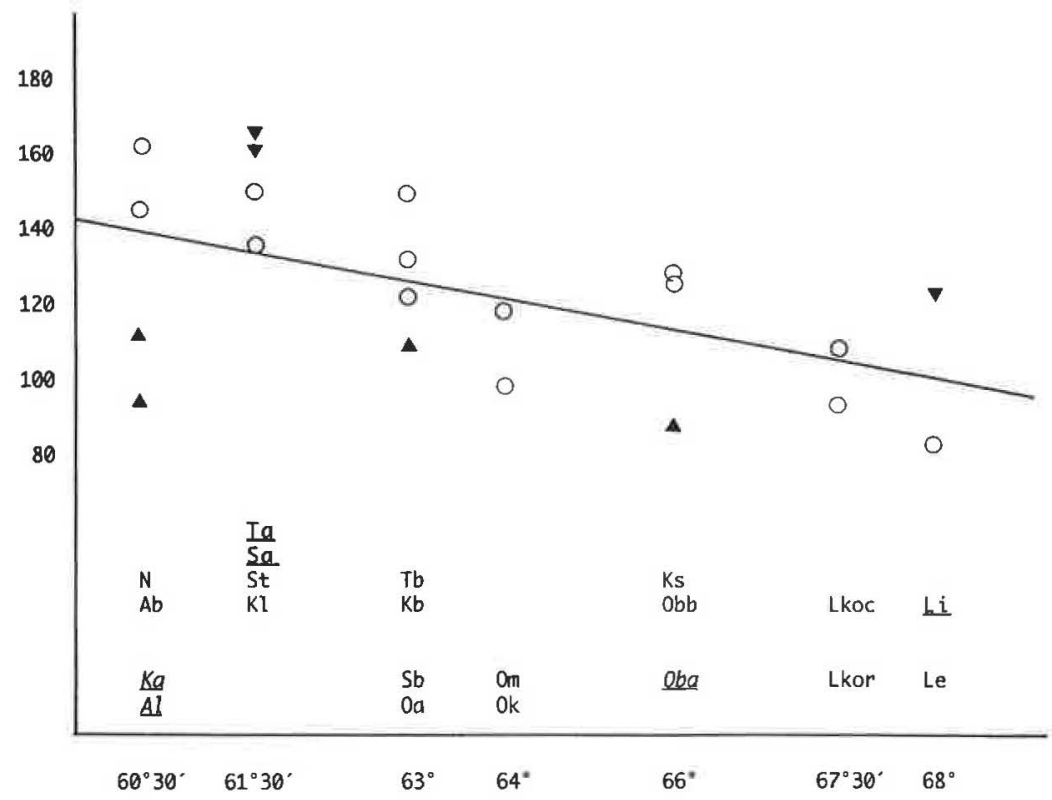

determine. Once again, we explain the phenomenon as a lack of knowledge. The remaining Limnephilus subcentralis has an extension from $\mathrm{Ok}$ to Lkor, a leap of $230 \mathrm{~km}$, and could even be a fardistance migrant. But we have never read a note of migration among Trichoptera. And even now, all evidence on migration is totally lacking.

In Fig. 1, we put the northern latitude of the weighted centre of Finnish biogeographical provinces - from $60^{\circ} 30^{\prime}$ to $68^{\circ} 30^{\prime}$ - and the sum of Trichoptera species found in them, and calculated a regression line. The correlation, $r=-0.824$ is very good and we claim that in the north of Finland about 50 species less is found than in the south. This trend seems to be relatively stable, as a comparison of the provinces with high level of trichopteran investigation gave roughly the same regression. When comparing the actual finds from the provinces with this regression line, we see that the three provinces with most incomplete investigation work are $\mathrm{Al}, \mathrm{Oba}$, and $\mathrm{Ka}$, in which the numbers of finds are $-32 \%,-21 \%$, and $-19 \%$ below the regression line, respectively. Perhaps also $\mathrm{Le},-17 \%, \mathrm{Oa},-16 \%$, and $\mathrm{Ok},-15 \%$ belong to the underachievers. On the other hand, the investigation of Trichoptera seems to be at a good level in $\mathrm{Ta},+23 \%, \mathrm{Li},+21 \%$, and in $\mathrm{Sa}$, $+20 \%$.

No regression was found in a graph at the east- west direction.

Finally there are 13 species with no new provincial finds, "numbers", during the last 37 years. These species seem to divide into three: those which are very rare and can be found in southern Finland only, like Orthotrichia tragetti, Oxyethira ecornuta, Holocentropus stagnalis, and Agrypnetes crassicornis; or are rare and can be found in the north only, like Agrypnetes crassicornis (again!), Ecclisopteryx dalecarlica, Grammotaulius sibiricus, Asynarchus impar, and Molanna nigra. The third group is formed by those, whose distribution ceases in the middle of Finland, in $\mathrm{Kb}, \mathrm{Om}$, or Obb: Hydroptila lotensis, Ecnomus tenellus, Trichostegia minor, Limnephilus auricula, and Ylodes detruncatus. In Finland we have no evidence of declining species among Trichoptera, but perhaps they could be found among these groups.

\section{Discussion}

The use of references and sources was standardized as much as possible and there was no intention to discriminate anybody. On the contrary, the last letters, etc., doubled much of the new finds and made thus our paper more reliable.

On provinces $\mathrm{Ka}$ and $\mathrm{Kl}$, the omission of finds from the area, which today belong to Russia, came 
somewhat late. It may be that we have not snapped up all substitutive finds. Let that be a partial explanation for low yield at $\mathrm{Ka}$ ( and $\mathrm{Kl},-12 \%$ ), and let us assume that the deficiency is corrected in near future. Provinces $\mathrm{Oa}, \mathrm{Om}, \mathrm{Oba}$, and $\mathrm{Obb}$ are all very flat, with few hills and no mountains. This may cause a monotonous profile of water bodies and thus explain the low numbers of finds. But why only at $\mathrm{Oa}$ and $\mathrm{Oba}$, when both $\mathrm{Om}$ and $\mathrm{Obb}$ are above the line? We stick to the hypothesis of a low investigation activity.

A quickly made division of Potamophylax "stellatus" was not easy. Obviously it would be worth of another work. As a summary, P. latipennis seems to occur everywhere, except in Al, but $P$. cingulatus is lacking in southernmost Finland and perhaps also in farthest NW-corner of Finland, Kilpisjärvi.

Most of the experts seem to have lots of undetermined material both in pin, in refrigerator, and in spirit. Thus we foresee much new information in near future. We are sure that even a haphazard collection of Trichoptera in those incompletely investigated provinces $\mathrm{Al}, \mathrm{Ka}, \mathrm{Oa}, \mathrm{Ok}, \mathrm{Oba}$, and Le would lead to new finds, too. Today, the small amount of experts capable of determining Trichoptera is a limiting factor in Finland. However, we are happy to report that a determination key to Finnish Caddisflies has been published in a book for fly fishers (Rinne et al. 1995). We recommend the book, although the key, originally constructed by late Jorma Kyrki at Oulu University, is published in a simplified form, which certainly causes difficulties in some critical genuses.

Addendum: During the editorial work of this paper, three interesting papers dealing with Trichoptera have been published in Finnish (Järventausta 1997, Mattila \& Salokannel 1997, Saarela 1997). They are put in as references $100-102$ at the end of reference list, and their data are included in the Table 1, but not discussed elsewhere.

Acknowledgements. Our thanks go to the following persons, who have given us valuable information: Antti Aalto, Heli Alanne, Anders Albrecht, Pauli Bagge, Mauri Hirvenoja, Erkki Huttula, Heikki Hämäläinen, Juhani Itämies, Sinikka Jokela, Kari Järventausta, Seppo Koponen, Esa Koskenniemi, Keijo Mattila, Jukka Mankki, Lauri Paasivirta, Risto Palomäki, Marja-Liisa Poikolainen, Eeva-Riitta Puomio, Esko Saarela, Juha Salokannel, Eino Savolainen, Antero Suoranta, Sirpa Takalo, Anne Tuorila, Harri Turunen and Kari-Matti Vuori. In addition to good ideas and fruitful discussions they have contributed to this work with at least 200 working hours.

\section{References}

1) Andersen, T. \& Wiberg-Larsen, P. 1987: Revised checklist of NW European Trichoptera. - Ent, scand. 18: 165-184.

2) Anttila, M.-E. 1985: Koskikivikoiden pohjaeläimistö Kyrönjoen vesistössä. (In Finnish). - National Board of Waters, Finland. Report 257. Helsinki. 72 pp.

3) Bagge, P. 1982: Saarijärven Pyhä-Häkin kansallispuiston ja sen lähiympäristön vesiperhoset (Trichoptera). (In Finnish). Pp. 52-55 in: Viitala, J. (Ed.) 1982: Saarijärven Pyhä-Häkin kansallispuiston ja sen lähiympäristön metsäjärvien veden laatu, klorofyllipitoisuus, eläinplankton, pohjaeläimistö sekä vesihyönteis- ja vesipunkkilajisto. - Jyväskylän yliopiston Biologian laitoksen Tiedonantoja 29. Jyväskylä. 63 pp.

4) Bagge, P. 1982: Caddis flies (Trichoptera) and water bugs (Heteroptera, Corixidae) of small water bodies caught by light trapping in southeastern Finland. - Notulae Entomol. 62: 73-81.

5) Bagge, P. 1987: Effects of pollution and eutrophication on the Trichopteran fauna of northem L. Päijänne and some adjacent larger lakes (Central Finland). - Biol. Res. Rep. Univ. Jyväskylä 10: 105-113.

6) Bagge, P. 1987: Emergence and distribution of Hydroptilidae in the littoral and outlet biocoenoses of lake Konnevesi (Central Finland). Pp. 337-341 in: Bournaud, M. \& Tachet, H. (Eds.) 1987: Proc. of the 5th Int. Symp. on Trichoptera. - Dr W. Junk Publishers. Dordrecht. 500 pp.

7) Bagge, P. 1992: Communities and habitats of filter feeding caddisflies in the lake outlet biocoenoses of Central Finland. Pp. 95-99 in: Tomaszewski C. (Ed.) 1992: Proc. of the 6th Symp. on Trichoptera. - Adam Mickiewicz University Press. Warsaw. 200 pp.

8) Bagge, P. 1995: Emergence and upstream flight of lotic mayflies and caddisflies (Ephemeroptera and Trichoptera) in a lake outlet, central Finland. - Entomol. Fennica 6: 91-97.

9) Bagge, P. 1995: Vesiperhosten (Trichoptera) ruokailukillat ja aikuisten emergenssi Konneveden rantavyöhykkeessä sekä Siikakoskessa. (In Finnish). Pp. 109-119 in: Valkeajärvi, P: (Ed.) 1995: Luonnontilan muutokset Konnevedessä; 25 vuotta vesiluonnon tutkimusta. Kalatutkimuksia Fiskundersökningar 100. - Riistanja kalantutkimus. Helsinki. 300 pp.

10) Bagge, P. \& Salmela, V.-M. 1978: The macrobenthos of the river Tourujoki and its tributaries (Central Finland). 1. Plecoptera, Ephemeroptera and Trichoptera. - Notulae Entomol. 58:159-168.

11) Bergman, G. \& Jansson, A. 1985: Accessions to the Zoological Museum, University of Helsinki/Helsingfors in 1984. - Memor. Soc. F. Fl. Fenn. 61: 138-139.

12) Brander, T. 1964: Lisiä Lounais-Hämeen hyönteisluet- 
teloihin (Odonata,Orthoptera, Neuroptera, Mecoptera ja Trichoptera). (In Finnish). — Lounais-Hämeen luonto 16: 69-72.

13) Esben-Petersen, P. 1916: Vaarfluer.(In Danish). G.E.C. Gads Forlag, Copenhagen.218 pp.

14) Hackman, W. 1964: Helsingin Yliopiston Eläinmuseon hyönteiskokoelmien kasvu toimintavuonna 1963-64. (In Finnish). - Mem. Soc. F. Fl. Fenn. 40: 213-215.

15) Hiilivirta, P. 1982: Tricholeiochiton fagesii (Guinard) (Hydroptilidae) new for Finland. - Notulae Entomol. 62: 154

16) Hiilivirta, P. \& Laasonen, E. 1984: Trichoptera. Pp. 24 27 in: Hulden, L. 1984: A check list of the Finnish insects. Small orders. - Notulae Entomol. 64: 1-29.

17) Hulden, L., Meinander, M., Nybom, O. \& Silfverberg, H. 1977: Deletions from the Finnish Fauna I. - Notulae Entomol. 57:11-12.

18) Huttunen, P. \& Hämäläinen, H. 1989: Purojen minimi$\mathrm{pH}:$ ennustaminen pohja-eläinten avulla. (In Finnish). - Vesi- ja Ympäristöhallinnon julkaisuja 31: 41-83.

19) Hämäläinen, H. 1992: Havaintoja Suomen uhanalaisista vesihyönteisistä. (In Finnish). — Kulumus 12: 33-35.

20) Jussila, R. 1966: Turun Eläin- ja Kasvitieteellisen Seuran Hyönteistieteellinen Kerho. (In Finnish). - Ann. Ent. Fenn. 32: 332-336.

21) Järventausta, K. 1994: Limnephilus luridus Curtis -vesiperhonen yleisenä eräillä Etelä-Hämeen soilla. (In Finnish). - Diamina 3: 16.

22) Katsalova, O. L. 1987: Trichoptera - Rutseiniki. (In Russian). Pp.107-193 in: Medvedeva, G. S. 1987: Opredelitelj nasekomyh Europeiskoi tsasti SSSR, Tom IV, shestaja tsastj. —Izdateljstvo "Nauka”, Leningrad. 200 pp.

23) Koskenniemi, E. 1994: Colonization, succession and environmental conditions of the macrozoobenthos in a regulated, polyhumic reservoir, western Finland. - Int. Revue ges. Hydrobiol. 79: 521-555.

24) Kuusela, K. 1979: Early summer ecology and community structure of the macrozoobenthos on stones in the Jäväjänkoski rapids on the river Lestijoki, Finland. Acta Univ. Ouluensis, Ser A 87, Biol. 6: 1-123.

25) Laasonen, E. \& Laasonen, L. 1987: Kolme Inarin Lapille uutta vesiperhoslajia. (In Finnish). - Notulae Entomol. 67: 215

26) Laasonen, E. \& Laasonen, L. 1988: Polycentropus irroratus (Curt.) (Polycentropodidae) Inarin Lapille uutena. (In Finnish). - Notulae Entomol. $68: 153$.

27) Lax, H.-G., Koskenniemi, E., Sevola, P. \& Bagge, P. 1993: Tenojoen pohjaeläimistö ympäristön laadun kuvaajana. (In Finnish). - Vesi- ja ympäristöhallinnon julkaisuja - sarja A 131. Helsinki. 124 pp.

28) Leivo, J. 1996: Limnephilus hirsutus (Pictet), Suomelle uusi vesiperhonen. (In Finnish). — Diamina 5: 28-30.

29) Macan, T. T. \& Worthington C. J. 1973: A key to the adults of the British Trichoptera. - Freshwater Biological Association, Scientific Publication No.28, Windermere. $151 \mathrm{pp}$.

30) Malicky, H. 1983: Atlas of European Trichoptera. Dr W. Junk Publishers, The Hague. 298 pp.

31) Mattila, K. 1994: Hyönteishavaintoja 1993. (In Finn- ish). - Diamina 3: 39-42.

32) Mattila, K. 1995: Hyönteistiedonannot 1994. (In Finnish). - Diamina 4: 40-42.

33) Mattila, K. 1996: Hyönteistiedonannot 1995. (In Finnish). - Diamina 5: 32-33.

34) Mettinen, A. 1994a: Mustionjoen ja Fiskarsinjoen makroskooppinen pohjaeläimistö ja sen muuttuminen vuodesta 1979. 24 pp. In: Jokinen, O.: Mustionjoen, Fiskarsinjoen, Pohjanpitäjänlahden ja Tammisaaren merialueen yhteistarkkailun pohjaeläintutkimukset vuodelta 1993. Pohjaeläimistön muuttuminen vuodesta 1979. (In Finnish). — Länsi-Uudenmaan vesi ja ympäristö r.y. Lohja, 40 pp. (ISSN 0789-9084).

35) Mettinen, A. 1994b: Porvoonjoen pohjaeläintutkimus kalataloudellisen yhteistarkkailun osana vuodelta 1992. 13 pp. In: Henriksson, M. \& Myllyvirta, T.: Porvoonjoen kalatalouden yhteistarkkailu. (In Finnish). — ItäUudenmaan ja Porvoonjoen vesien- ja ilmansuojeluyhdistys. Porvoo, $33 \mathrm{pp}$.

36) Muotka, T. 1993: Microhabitat use by predaceous stream insects in relation to seasonal changes in prey availability. - Ann. Zool. Fennici 30: 287-297.

37) Nybom, O. 1960: List of Finnish Trichoptera. — Fauna Fennica, Helsinki. 56 pp.

38) Nybom, O. 1964: En för Finlands fauna ny nattslända. (In Swedish). - Notulae Entomol. 44: 52.

39) Nybom, O. 1972: The invertebrate fauna of the Kilpisjärvi area, Finnish Lapland. 13. Trichoptera. - Acta Soc. F. Fl. Fenn, 80: 181-188.

40) Nybom, O. 1980: Några för Finland nya arter av Trichoptera.(In Swedish). — Notulae Entomol. 60: 197-198.

41) Nybom, O. 1983: Oxyethira klingstedtii sp.n. (Trichoptera, Hydroptilidae) from Finland. - Notulae Entomol. 63: 65-66.

42) Nybom, O. 1984: Trichoptera of Inari Lapland, with special reference to light trap material. - Kevo Notes 7: 59-66.

43) Nybom, O. \& Brander, T. 1963: Lounais-Hämeen vesiperhosista, Trichoptera. (In Finnish). - Lounais-Hämeen luonto 14: 79-82.

44) Nyman, C., Anttila, M.-E. \& Lax, H.-G. 1986: Koskien pohjaeläimistö jokien laatuluokittelun perustana. (In Finnish). - Vesi- ja ympäristöhallinnon julkaisuja 3. Helsinki 76 pp.

45) Paasivirta, L. 1976: Suomunjärven (Lieksa) pohjaeläimistön koostumus, biomassa ja tuotanto. (In Finnish). - Joensuun korkeakoulu, Karjalan tutkimuslaitos, Julkaisuja N:o 18. Joensuu, 16 pp.

46) Paasivirta, L. 1983: Konneveden vesihyönteisistä. (In Finnish). - Jyväskylän yliopiston Biologian laitoksen Tiedonantoja 34: 25-36.

47) Paavola, R., Kuusela, K. \& Itämies, J. 1994: Vuotoksen suunnitellun allasalueen luonnon tutkimukset. Osa K: Vesien pohjaeläimet. — Tutkimusraportti Kemijoki Oy:lle, Oulun yliopisto, 17 ss.

48) Peltonen, O. 1981: Kesäretki Kuusamoon 1979-07-0106. (In Finnish). - Notulae Entomol. 61: 217.

49) Rinne, A., Vainio, J., Huitila, M \& Soine J. 1995: Perhokalastus ja vesiperhoset. (In Finnish), — Werner Söder- 
ström Oy, Porvoo, 253 pp. ISBN 951-0-20507-9.

50) Siitonen, O. 1980: Vesiperhosia (Limnophilidae) Ahvenanmaalta. (In Finnish). - Notulae Entomol. 60: 227.

51) Silfverberg, H. 1981: Additions to the Finnish insect fauna during the years 1976-1980. Notulae Entomol. 61: 45-61.

52) Silfverberg, H. 1986: Additions to the Finnish insect fauna during the years 1981-1985. - Notulae Entomol. 66: $131-152$.

53) Sundell, P., Granberg, K. \& Hynynen, J, 1994: Keiteleen kanavan rakentamisen vaikutukset vesistön veden laatuun, eliöstöön ja kalatalouteen vuosina 1990-93. (In Finnish). - Ympäristöntutkimuskeskus, Jyväskylän Yliopisto, Jyväskylä 62 pp.

54) Svensson, B. W. \& Tjeder B. 1975 : Check-list of the Trichoptera of north-western Europe. - Ent. scand. 6: 261-274.

55) Valtonen, P. 1993: Hyönteistieteellisiä tiedonantoja. (In Finnish). - Diamina 2: 36-39.

56) Vuori, K.-M. 1993: Kruunupyynjoen koskien pohjaeläimistö.(In Finnish). Vaasan vesi- ja ympäristöpiiri. - Vaasa. 15 pp.

57) Vuori, K.-M. \& Joensuu, I. 1996: Impact of forest drainage on the macroinvertebrates of a small boreal headwater stream. Do buffer zones protect lotic biodiversity? - Biol. Conservation 77: 87-95.

58) Vuori, K.-M. \& Parkko, M. 1996: Assessing pollution of the river Kymijoki via hydropsychid caddis flies: population age structure, microdistribution and gill abnormalities in the Cheumatopsyche lepida and Hydropsyche pellucidula larvae. - Arch. Hydrobiol. 136: 171-190.

59) Wallace, I. D. 1991: A review of the Trichoptera of Great Britain. Research \& survey in nature conservation No.32. - Nature Conservancy Council, Peterborough. 61 pp. ISBN 0861396936.

\section{Other sources: Manuscripts, Collec- tions, and Letters}

72) Heikura, R. 1986: Valorysällä saatujen vesiperhosten ekologiasta Värriön luonnonpuistossa vuosina 1978 ja 1979. (In Finnish). — Oulu University. 131 pp. (A manuscript for the grade of Bachelor in Science).

60) Huttula, E. 1966: Kunnostettujen koskien kalakantojen seuranta, Lapin ympäristökeskuksen tutkimusprojekti. (In Finnish). - Lapin Ympäristökeskus. (A manuscript).

61) Hyytiäinen, U.-M. 1990: Salonpuron perkauksen vaikutus Lestijärven pohjaeläimistöön, lähtötilanteen selvitys. (In Finnish). - Kokkolan vesi- ja ympäristöpiiri. 11 pp. (A manuscript).

62) Krooks, K. 1984: Pohjaeläimistöstä Kalajoen suvannoissa Hamarinkosken alapuolella keväällä 1984. (In Finnish). - Kokkolan vesipiirin vesitoimisto. 15 pp. (A manuscript).

63) Laasonen, P. 1995: Koillismaan jokien vesiperhoshavaintoja (In Finnish). - Oulu University. (A manu- script).

64) Lax, H.-G. 1989: Bottenfauna i forsarna i Lappfjärds ås nedre del och i Bötom å. (In Swedish). — Vatten- och miljöstyrelsens duplikatserie nr 141, 25 pp. (A manuscript).

65) Luotonen, H. 1988: Lestijoen koskialueiden pohjaeläimistö.(In Finnish). — Oulu University, Department of Zoology. 25 pp. (A manuscript).

66) Nyman, C. 1993: Perhonjoen keskiosan säännöstelyn vaikutuksia koskien pohjaeläimistöön. (In Finnish). Kokkolan vesi- ja ympäristöpiiri. 28 pp. (A manuscript).

67) Paasivirta, L. 1993: Aurajoen Nautelankosken pohjaeläimistö. (In Finnish). - Valtion kalatalousoppilaitos, Parainen. $17 \mathrm{pp}$. (A course for students).

68) Paasivirta, L. 1996: Limnologian kesäkurssi, bioindikaattorit, opisto 2, 26.-29. 8. 1996. (In Finnish). - Valtion kalatalousoppilaitos, Parainen. 26 pp. (A course for students).

69) Puska, M. 1993: Merikarvianjoen Lankosken pohjaeläimistö ja sen merkitys kalojen ravintona. (In Finnish). - Valtion kalatalousoppilaitos, Parainen. 17 pp. (A manuscript for the grade).

70) Riihelä, M.1994: Elimysalon järvien pohjaeläintutkimus. (In Finnish). - Vesi- ja ympäristöhallituksen monistesarja nro 558, $55 \mathrm{pp}$. (A manuscript).

71) Valovirta, M. 1966: Eräiden Leppävedellä (PH) tavattavien vesiperhostoukkien (Trichoptera) ekologisesta erilaistumisesta. (In Finnish). - Helsinki University. $85 \mathrm{pp}$. (A manuscript of a thesis for the grade of Bachelor in Science).

72) Heikura, R. 1986: Valorysällä saatujen vesiperhosten ekologiasta Värriön luonnonpuistossa vuosina 1978 ja 1979. (In Finnish). - Oulu University.131 pp. (A manuscript for the grade of Bachelor in Science).

80) Coll. Finnish Museum of Natural History, Zoological Museum, Helsinki University, Helsinki

81) Coll. Zool, Mus. Turku University, Turku

82) Coll. Zool. Mus. Oulu University, Oulu

83) Coll. Museum of Natural History in Kuopio, Kuopio

84) Coll. Lounais-Hämeen luonnonsuojeluyhdistys (Association for Nature Protection in Southwestern Häme), Forssa

85) Coll. H. Hämäläinen, Joensuu

86) Coll. E. \& L. Laasonen, Helsinki

87) Coll. M. \& T. Mutanen, Rovaniemi

88) Coll. and archives O. Nybom, Helsinki

89) Coll. H. Turunen, Tampere

90) Albrecht, A.: Letter 14.10.1996.

91) Hirvenoja, M.: Letter 28.10 .1996

92) Järventausta, K.: Letter 15.10.1996.

93) Saarela, E.: Letters 14.10., 04.12.1996, and 30.1.1997.

94) Salokannel, J.: Letters 10.10., 13.11., and 20.11.1996.

\section{Addendum:}

100) Järventausta, K. 1997: Kuusamon ja Lapin vesiperhosiin tutustumassa. (In Finnish), — Diamina 6: 17-29. 
101) Mattila, K. \& Salokannel, J. 1997: Parikkalan Siikalahden vesiperhosista. (In Finnish). - Diamina 6: 30-35.
102) Saarela, E. 1997: Crunoecia irrorata (Curtis), Suomelle uusi vesiperhoslaji. (In Finnish). — Diamina 6: 13-14. 
Table 1: The finds of trichopteran species in Finnish biogeographical provinces. Symbol - means that the species is not found in the respective province. Symbol + means a find reported already in Nybom 1960. New finds after that are shown with a number, which also reveals the reference. If the find data is unpublished, it is presented in Appendix under the same number.

\begin{tabular}{lllllllllllllllllllll}
\hline Al & Ab & $\mathrm{N}$ & $\mathrm{Ka}$ & $\mathrm{St}$ & $\mathrm{Ta}$ & $\mathrm{Sa}$ & $\mathrm{Kl}$ & $\mathrm{Oa}$ & $\mathrm{Tb}$ & $\mathrm{Sb}$ & $\mathrm{Kb}$ & $\mathrm{Om}$ & $\mathrm{OK}$ & Oba & Obb & $\mathrm{Ks}$ & Lkoc & Lkor & Le & Li
\end{tabular}

\section{RHYACOPHILIDAE}

Rhyacophila Pictet, 1834 nubila (Zetterstedt, 1840)

fasciata Hagen, 1859

septentrionalis McLachlan, 1865

obliterata McLachlan, 1863

\section{GLOSSOSOMATIDAE}

Glossosoma Curtis, 1834 nylanderi McLachlan 1879

Agapetus Curtis, 1834

ochripes Curtis, 1834

\section{HYDROPTILIDAE}

Agraylea Curtis, 1834

multipunctata Curtis, 1834

cognatella McLachlan, 1880 sexmaculata Curtis, 1834 pallidula McLachlan, 1875

Hydroptila Dalman, 1819 sparsa Curtis, 1834 simulans Mosely, 1920 lotensis Mosely, 1930 cornuta Mosely, 1922 angulata Mosely, 1922 occulta (Eaton, 1873)

kimminsi Mosely, 1930 tineoides Dalman, 1819 pulchricomis Pictet, 1834 pulchicomis Pictet, 1834

forcipata (Eaton, 1873) lamellaris Eaton, 1873

Orthotrichia Eaton, 1873 tragetti Mosely, 1930 costalis (Curtis, 1834) tetensii Kolbe, 1887

\begin{tabular}{|c|c|c|c|c|c|c|c|c|c|c|c|c|c|c|c|c|c|c|c|c|}
\hline+ & + & + & + & + & + & + & + & + & 46 & + & - & + & 80 & + & 92 & 88 & - & - & - & - \\
\hline - & - & - & - & - & 84 & - & - & - & 6 & - & - & - & + & + & + & + & 86 & 92 & + & + \\
\hline- & 80 & 80 & 55 & 55 & + & + & - & - & 6 & - & - & - & - & - & - & - & - & - & - & - \\
\hline - & 82 & + & - & + & - & + & + & - & 46 & - & - & - & - & + & - & - & - & - & - & - \\
\hline- & - & + & - & + & 32 & + & - & - & + & - & - & - & - & + & + & - & - & - & - & - \\
\hline - & - & - & - & - & - & - & - & - & + & - & - & + & - & - & + & - & - & - & - & - \\
\hline- & + & + & - & + & + & + & + & + & 6 & - & + & + & 88 & - & + & - & + & - & - & 86 \\
\hline- & + & + & - & + & - & + & - & + & 6 & - & - & 88 & - & - & + & - & - & - & - & - \\
\hline- & - & - & - & 31 & - & + & + & - & - & - & - & - & - & - & - & - & - & - & - & - \\
\hline+ & + & + & 86 & + & + & + & + & + & + & + & + & + & - & 82 & 80 & 82 & 80 & 91 & + & + \\
\hline+ & + & + & - & 82 & + & + & + & - & 6 & 88 & + & + & + & 88 & + & - & - & - & - & - \\
\hline- & 82 & + & - & - & - & - & + & - & 6 & - & - & - & - & - & - & - & + & - & - & + \\
\hline - & - & + & - & + & 80 & + & + & + & 6 & 88 & 18 & 24 & 63 & 88 & + & + & + & - & + & 85 \\
\hline- & - & - & - & - & - & + & - & - & - & - & - & - & - & - & - & - & - & - & - & - \\
\hline - & + & + & - & + & 93 & + & 82 & - & + & - & + & 61 & - & - & - & - & - & - & - & - \\
\hline
\end{tabular}


costalis auct. nec. Curtis, 1834

tristella Klapálek, 1895

simplex Ris, 1897

frici Klapálek, 1890

falcata Morton, 1893

distinctella McLachlan, 1880

sagittifera Ris, 1897

ecornuta Morton, 1893

klingstedti Nybom, 1983

mirabilis (Morton, 1904)

Stactobiella Martynov, 1924

risi (Felber, 1908)

ulmeri (Siltala, 1908)

Tricholelochiton Kloet \& Hincks, 1944

fagesii (Guinard, 1879)

\section{PHILOPOTAMIDAE}

\section{Philopotaminae}

Philopotamus Stephens, 1829

montanus (Donovan, 1813)

Wormaldia McLachlan, 1865

Subnigra McLachlan, 1865

Chimarrinae

Chimarra Stephens, 1829

marginata (Linnaeus, 1767)

\section{PSYCHOMYIIDAE}

Tinodes Curtis, 1834

waeneri (Linnaeus, 1758)

Lype McLachlan, 1878

phreopa (Stephens, 1836)

phaeopa (Slephens, 1836 )

Psychomyia Latreille, 1829

pusilla (Fabricius, 1781)

\section{ECNOMIDAE}

Ecnomus McLachlan, 1864 tenellus (Rambur, 1842) 


$\begin{array}{llllllllllllllllllllllll}\text { Al } & \text { Ab } & \mathrm{N} & \mathrm{Ka} & \mathrm{St} & \mathrm{Ta} & \mathrm{Sa} & \mathrm{KI} & \mathrm{Oa} & \mathrm{Tb} & \mathrm{Sb} & \mathrm{Kb} & \mathrm{Om} & \mathrm{Ok} & \mathrm{Oba} & \mathrm{Obb} & \mathrm{Ks} & \mathrm{LkOC} & \mathrm{Lkor} & \mathrm{Le} & \mathrm{Li}\end{array}$

\section{POLYCENTROPODIDAE}

Neureclipsis McLachian, 1864 bimaculata (Linnaeus, 1758)

Plectrocnemia Stephens, 1836 Plectrocnemiella Mosely, 1934 conspersa (Curtis, 1834) carelica (Nybom, 1950) conjuncta Martynov, 1914

Polycentropus Curtis, 1835

flavomaculatus (Pictet, 1834)

multiguttatus (Curtis, 1835)

irroratus (Curtis, 1835)

multiguttatus McLachlan, 1878

Holocentropus McLachlan, 1878 dubius (Rambur, 1842)

picicornis (Stephens, 1836)

picicomis (Stephens, 1836)

insignis Martynov, 1924

stagnalis (Albarda, 1874)

Cyrnus Stephens, 1836

insolutus McLachlan, 1878

trimaculatus (Curtis, 1834)

fennicus Klingstedt, 1937

crenaticornis (Kolenati, 1859)

flavidus McLachlan, 1864

\section{HYDROPSYCHIDAE}

Hydropsyche Pictet, 1834 pellucidula (Curtis, 1834) saxonica McLachlan, 1884 siltalai Dōhler, 1963

instabilis auct. nec. (Curtis, 1834)

angustipennis (Curtis, 1834)

contubernalis McLachlan, 186

borealis Martynov, 1926

ornatula auct. nec. McLachlan, 1878

bulgaromanorum Malicky, 1977

guttata auct. nec. Pictet, 1834

Ceratopsyche Ross \& Unzicker, 1977 nevae (Kolenati, 1858)

silfvenii (Ulmer, 1906)

$\begin{array}{cccccccc}31 & 82 & + & + & 69 & + & + & + \\ - & 20 & - & - & - & + & + & + \\ + & + & + & + & 69 & + & + & + \\ + & + & + & + & + & + & + & + \\ - & - & 88 & + & + & - & + & + \\ & & & & & & & \\ - & - & - & - & 40 & 40 & 40 & +\end{array}$

$+$

$\begin{array}{ll}+ & + \\ 2 & 8 \\ 2 & + \\ + & + \\ 64 & +\end{array}$

88
-
+
+
+
+

$\begin{array}{ll}88 & + \\ - & 18 \\ + & + \\ + & + \\ - & \end{array}$

+
+
+
+
+
83

24
66
+
+
+
+

88
-
88
80
+

80
+
+
9
9
9
-
-
+
+

\begin{tabular}{|c|c|c|}
\hline 80 & 91 & + \\
\hline - & - & - \\
\hline 100 & 80 & + \\
\hline+ & + & + \\
\hline
\end{tabular}


Cheumatopsyche Wallengren, 1891

lepida (Pictet. 1834)

\section{ARCTOPSYCHIDAE}

Arctopsyche McLachlan, 1868 ladogensis (Kolenati, 1859)

\section{PhRYganedoAe}

Trichostecia Kolenati, 1848 minor (Curtis, 1834)

\section{Agrypnia Curtis, 1835} pagetana Cuitis, 1835 picta Kolenat, 1848 obsoleta (Hagen, 1864) sahlbergi (McLachlan, 1880) principalis (Martynov, 1909) czerskyi (Martynov, 1924)

Agrypnetes.McLachlan, 1876 crassicomis McLachlan, 1876

\section{Phryganea Linnaeus, 1758} grandis Linnaeus, 1758 bipunctata Retzius, 1783

striata auct nec. Linnaeus, 1758

Oligotricha Rambur, 1842 lapponica (Hagen, 1864) striata (Linnaeus, 1758) ruficrus (Scopoli, 1763)

Hagenella Martynov, 1924 clathrata (Kolenati, 1848)

Oligostomis Kolenati, 1848 reticulata (Lirnaeus, 1761)

Semblis Fabricius, 1775

Holostomis Mannerheim, 1838 phalaenoides (Linnaeus, 1758) atrata (Gmelin, 1790)

\section{BRACHYCENTRICAE}

Brachycentrus Curtis, 1834 subnubilus Curtis, 1834 


$\begin{array}{lllllllllllllllllllllllll}\text { Al } & \text { Ab } & \mathrm{N} & \mathrm{Ka} & \mathrm{St} & \mathrm{Ta} & \mathrm{Sa} & \mathrm{Kl} & \mathrm{Oa} & \mathrm{Tb} & \mathrm{Sb} & \mathrm{Kb} & \mathrm{Om} & \mathrm{Ok} & \text { Oba } & \text { Obb } & \mathrm{Ks} & \text { Lkoc } & \text { Lkor } & \text { Le } & \mathrm{Li}\end{array}$

Micrasema McLachlan, 1876

gelidum McLachlan, 1876

setiferum (Pictet, 1834)

nigrum (Brauer, 1857)

\section{LEPIDOSTOMATIDAE}

Crunoecia McLachlan, 1876 irrorata (Curtis, 1834)

Lepidostoma Rambur, 1842 hirtum (Fabricius, 1775)

\section{APATANIIDAE}

Apatania Kolenati, 1848

wallengreni McLachlan, 1871 stigmatella (Zelterstedt, 1840)

hispida (Forsslund, 1930)

auricula (Forsslund, 1930)

zonella (Zetterstedt, 1840)

dalecarlica (Forsslund, 1942)

kaisilai auct. nec. Forsslund

stylata Navás, 1916

\section{LIMNEPHILIDAE}

\section{Drusinae}

Ecclisopteryx Kolenati, 1848

dalecarlica Kolenati, 1848

guttulata auct. nec. Pictet, 1834

Limnephilinae

Limnephilus Leach, 1815

Limnophilus auct.

Limomict.

Thombicus (Linnaeus, 1758)

Havicomis (Fabriclus, 1787)

subcentrals Brauer, 1857

borealis (Zetterstedt, 1840)
femoratus (Zetterstedt, 1840)

mamnoratus Curtis, 1834

politus McLachlan, 1865

stigma Curtis, 1834

binotatus Curtis, 1834

xanthodes McLachlan, 1873

decipiens (Kolenati, 1848)

\begin{tabular}{|c|c|c|c|c|c|c|c|c|c|c|c|c|c|c|c|c|c|c|c|}
\hline- & + & - & - & 31 & + & - & - & 2 & + & + & 18 & + & 18 & - & + & + & + & 86 & + \\
\hline- & - & - & - & - & - & - & - & 2 & 8 & - & - & 24 & + & - & + & + & + & 47 & + \\
\hline
\end{tabular}


externus Hagen, 1861

sericeus (Say, 1824)

despectus Walker, 1852

luridus Curtis, 1834

quadratus Martynov, 1914

nebulosus Kirby, 1837

ignavus McLachlan, 1865

pantodapus McLachlan, 1875

fuscinervis (Zetterstedt, 1840)

elegans Curtis, 1834

griseus (Linnaeus, 1758)

hirsutus (Pictet, 1834)

dispar Mclachlan, 1875

fenestratus (Zetterstedt, 1840)

bipunctatus Curtis, 1834

subnitidus McLachlan, 1875

scalenus Wallengren, 1879

affinis Curtis, 1834

centralis Curtis, 1834

sparsus Curtis, 1834

auricula Curtis, 1834

vittatus (Fabricius, 1798)

extricatus McLachlan, 1865

nigriceps (Zetterstedt, 1840) fuscicornis (Rambur, 1842) algosus (McLachlan, 1868) diphyes (McLachlan, 1880) coenosus Curtis, 1834

Colpotaulius Kolenati, 1848

incisus (Curtis, 1834) major Martynov, 1909

Ironoquia Banks, 1916 dubia (Stephens, 1837)

Grammotaulius Kolenati, 1848 nigropunctatus (Retzius, 1783) atomarius (Fabricius, 1793)

strigosus (Curtis, 1834) sibiricus McLachlan, 1874 signatipennis McLachlan, 1876

Glyphotaelius Stephens, 1837 pellucidus (Retzius, 1783) 


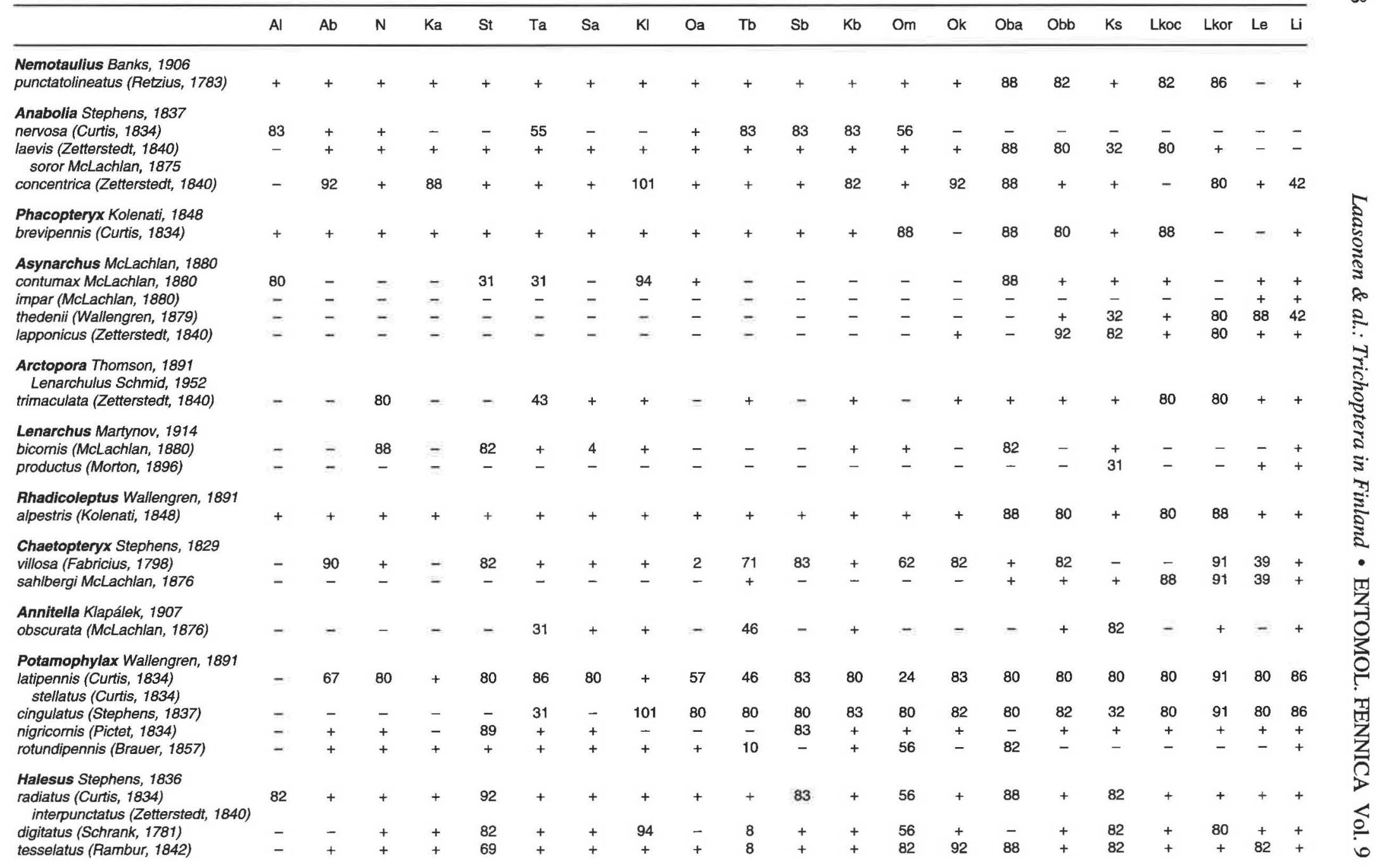


Micropterna Stein, 1874 lateralis (Stephens, 1837) sequax McLachlan, 1875

Hydatophylax Wallengren, 1891 infumatus (McLachlan, 1865)

Chilostigma McLachlan, 1876 sieboldi McLachlan, 1876

Brachypsyche Schmid, 1952 sibirica (Martynov, 1924)

\section{GOERIDAE}

Goera Stephens, 1829 pilosa (Fabricius, 1775)

Silo Curtis, 1830

pallipes (Fabricius, 1781)

\section{BERAEIDAE}

Beraea Stephens, 1833 pullata (Curtis, 1834)

Beraeodes Eaton, 1867 minutus (Linnaeus, 1761)

\section{SERICOSTOMATIDAE}

Sericostoma Latreille, 1825 personatum (Kirby \& Spence1826)

Notidobia Stephens, 1829 ciliaris (Linnaeus, 1761)

\section{MOLANNIDAE}

Molanna Curtis, 1834

angustata Curtis, 1834

albicans (Zetterstedt, 1840)

nigra (Zetterstedt, 1840)

carbonaria McLachlan, 1877 submarginalis McLachlan, 1872

Molannodes McLachlan, 1866 tinctus (Zetterstedt, 1840)

$+$

$\begin{array}{lllllll}+ & + & + & + & + & + & + \\ - & - & + & + & + & + & + \\ - & - & - & - & - & - & + \\ + & + & + & + & + & + \\ - & + & + & + & + & +\end{array}$




$\begin{array}{lllllllllllllllllllllll}\text { Al } & \mathrm{Ab} & \mathrm{N} & \mathrm{Ka} & \mathrm{St} & \mathrm{Ta} & \mathrm{Sa} & \mathrm{Kl} & \mathrm{Oa} & \mathrm{Tb} & \mathrm{Sb} & \mathrm{Kb} & \mathrm{Om} & \mathrm{Ok} & \mathrm{Oba} & \mathrm{Obb} & \mathrm{Ks} & \text { Lkoc } & \text { Lkor } & \text { Le } & \mathrm{Li}\end{array}$

\section{LEPTOCERIDAE}

Ceraclea Stephens, 1829 nigronervosa (Retzius, 1783) fulva (Rambur, 1842)

senilis (Burmeister, 1839)

alboguttata (Hagen, 1860)

annulicornis (Stephens, 1876)

perplexe (Mclachen, 1877)

perplexe (McLach an, 1877)

dissimilis (Stephens, $18 \mathrm{c}$

Athripsodes Billberg, 1820 aterrimus (Stephens, 1876) aterrimus (Stephens, 1876)
cinereus (Curtis, 1834)

albifrons (Linnaeus, 1758)

albifrons (Linnaeus, 1758)

Mystacides Berthold, 1827

niger (Linnaeus, 1758)

azureus (Linnaeus, 1761)

longicomis (Linnaeus, 1758)

Triaenodes McLachlan, 1865

bicolor (Cuntis, 1834)

unanimis McLachlan, 1877

Ylodes Mine, 1934

reuteri (McLachlan, 1880)

simulans (Tjeder, 1929)

forsslundi (Tjeder, 1941)

detruncatus (Martynov, 1924)

\section{Erotesis McLachlan, 1877} baltica McLachlan, 1877

\section{Oecetis McLachlan, 1877} ochracea (Curtis, 1825)

furva (Rambur, 1842)

lacustris (Pictet 1834)

notata (Rambur, 1842)

(testacea (Curtis, 1834)

Leptocerus Leach, 1815

tineiformis Curtis, 1 


\section{Appendix}

The unpublished finds new for the biogeographical provinces are presented here as complete as possible:

72) Heikura, R. (see below).

60) Huttula, E. (1966, a manuscript). Psychomyia pusilla Lkor: Sodankylä Raudanjoki Kurttiokoski 8.7.1994 E.H. leg.

61) Hyytiäinen, U.-M. (1990, a manuscript). Ortotrichia costalis Om: Lestijärvi Vehkalahti 7.-9.6.1989 U.-M. H. leg.

62) Krooks, K. (1995, a manuscript). 3 finds, all K.K. leg., Limnephilus lunatus Om: Alavieska Kalajoki 21.5.1985, Chaetopteryx villosa Om: Kalajoki Jokisuu 14.5. 1984, Hydatophylax infumatus Om: Kalajoki 16.5.1984.

63) Laasonen, P. (1995, a manuscript). 6 finds, all P.L. leg. Rhyacophila obliterata Ok: Kuhmo 7103:6520 Korkea, Kiekinjoki 1995, Ithytrichia lamellaris Ok: Kuhmo 7102:6515 Korkea, Kesselinjoki 1995, Ceratopsyche silfvenii Ok: Kuhmo 7103:6520 Korkea, Kiekinjoki 1995, Silo pallipes Obb: Pudasjärvi 7305:5220 Sarajärvi Kouvajoki, Notidobia ciliaris Ok: Kuhmo 7102:6515 Korkea, Kesselinjoki 1995 and Obb: Pudasjärvi 7310: 5220 Sarajärvi Livojoki.

64) Lax, H.- G. (1989, a manuscript). 2 finds, both Oa: Karijoki and H.-G.L. leg. Hydropsyche contuberalis Lapväärtinjoki Ohriluoma 10.12.1986, Beraeodes minutus Kirkonkylä 5.11.1986.

65) Luotonen, H. (1988, a manuscript). 4 finds, all H.L. leg. Rhyacophila fasciata Om: Kannus Lestijoki Jäväjänkoski 6.1987, Glossosoma intermedia Om: Lestijärvi Lestijoki Jatkonkoski 6.1987, Philopotamus montanus Om: Kannus Lestijoki Jäväjänkoski 6.1987, Mystacides niger Om: Toholampi Lestijoki Kallisenkoski 8.1987.

66) Nyman, C. (1993, a manuscript). Hydropsyche saxonica Om: Kokkola Perhojoki Skatafors 5.1992 C.N. leg.

67) Paasivirta, L. (1993, a manuscript). Potamophylax latipennis Ab: Lieto Aurajoen Nautelankosken Myllykoski 4.5.1993 L.P. leg.

68) Paasivirta, L. (1996, a manuscript). Leptocerus tineiformis Ab: Turku Littoistenjärvi 24.1.1994 L.P. leg.

69) Puska, M. (1993, a manuscript). 4 finds, all St.: Merikarvia Lankoski and M.P. leg., Oxyethira frici 15.4.1992, Hydropsyche pellucidula 26.5.1992, H. siltalai 15.4.1992, Halesus tesselatus 15.4.1992.

70) Riihelä, M. (1994, a manuscript). Cyrnus flavidus Ok: Kuhmo Elimyssalo Ristonlampi 31.5-3.6.1993 M.R.leg.

71) Valovirta, M. (1966, a manuscript). Chaetopteryx villosa Tb: Laukaa Leppävesi 1960-1962 M.V. leg.

72) Heikura, R. (1986, a manuscript). 3 finds, all from Ks: Salla 752:61 Värriö and R. H. leg., Holocentropus dubius 14.-20.7.1979, Limnephilus flavicornis 29.30.8.1978, L. vittatus 5.8.-12.9.1978.

80) Coll. Zoological Museum, Helsinki University, Helsinki. 152 finds. Rhyacophila nubila Oba: Oulu, Vuoren- taus leg., Agraylea multipunctata Ok: Suomussalmi, W. Hellen leg., A. sexmaculata Ab: Pargas, Reuter leg. and N: Helsinge, Palmen leg., Hydroptila tineoides Obb: Kemin mlk. Karihaara 30.7.1942 A. Saarinen leg. and Lkoc: Muonio, J. Sahlberg leg., Ithytrichia lamellaris Ta: Lammi 19.8.1959 J. Kaisila leg., Oxyethira tristella $\mathrm{N}$ : Askola 16.7.1969 T. Suomalainen leg. and Ka: Vehkalahti, L. Tiensuu leg., Stactobiella risi Sa: Ruokolahti 25.6.1940 O. Nybom leg., Philopotamus montanus Ka: Vehkalahti, L. Tiensuu leg., Psychomyia pusilla Oba: Uleåborg, Coll. Nylander, Neureclipsis bimaculata Lkoc: Muonio, W. Hellen leg., Plectrocnemia conspersa St: Vammala 31.7.1970 K. Lahtivirta leg,, Obb: Pisavaara naturpark 17.7.1950 N.E.Saris leg., and Lkoc: Muonio 1.7.1948 J. Kaisila leg., Polycentropus flavomaculatus Obb: Kemin mlk 4.8.1942 Karihaara A. Saarinen leg. and Lkor: Pelkosenniemi R. Frey leg., Holocentropus picicornis Oba: Uleåborg, Coll Nylander and Lkoc: Muonio 14.7.1962 G. Roos leg., Cyrnus trimaculatus Ok: Sotkamo 25.7.1954 A. Mikkola leg., C. crenaticornis $\mathrm{N}$. Ekenäs $\mathrm{lk}$. Tvärminne 5.9.1928 Klingstedt leg., Hydropsyche angustipennis Oba: Uleåborg, Coll. Nylander, Obb: Rovaniemi 27.6.1929 H. Lindberg leg., and Lkoc: Muonio, W.Hellen leg., Arctopsyche ladogensis Oba: Oulu, Vuorentaus leg., Obb: Kemijärvi 9.7.1951 J. Carpelan leg., and Lkoc: Muonio 25.6.1925 J. Montell leg., Agrypnia pagetana Oba: Hailuoto 11.7.1966 J. Kaisila leg., A. picta Lkoc: Muonio 14.7.1962 G. Roos leg., A. obsoleta Oba: Oulu, Vuorentaus leg., Obb: Pisavaara nationalpark 20.7.1950 N.E. Saris leg., and Lkoc: Muonio 14.7.1962 G. Roos leg., Phryganea bipunctata Obb: Torneå 4.7.1924 H. Lindberg leg., Oligotricha lapponica Oba: Hailuoto, Vuorentaus leg., Lkoc: Muonio 734:36 26.6.1976 J. Jalava leg., and Lkor: Sodankylä Mutenia 1.7.1949 J. Kaisila leg., Oligostomis reticulata Lkoc: Kittilä Pallasjärvi 12.7.1951 J. Kaisila leg and Lkor. Sodankylä, Euren leg., Brachycentrus subnubilus Ka: Vehkalahti 6.6.1964 L. Tiensuu leg., Lepidostoma hirtum Obb: Pisavaara naturpark 14.7.1950 N.E.Saris leg., Apatania wallengreni Oba: Oulu, Vuorentaus leg., Obb: Kemijärvi 7.7.1951 J. Carpelan leg., Lkoc: Muonio 14.7.1971 E. Suomalainen leg, and Lkor: Sodankylä Tähtelä 6.6.1969 E. Suomalainen leg., A. stigmatella Obb: Turtola, J. Sahlberg leg., Lkoc: Muonio 11.8. 1972 E. Suomalainen leg, and Lkor: Saariselkä, B. Poppius leg., Limnephilus rhombicus Obb: Rovaniemen mlk Pisa 15.7.1950 H. Lindberg leg., Lkoc: Muonio, Silfvenius leg., and Lkor: Sodankylä Mutenia 1.7.1949 J. Kaisila leg., L. borealis Lkoc: Muonio, J. Montell leg. and Lkor: Saariselkä, B. Poppius leg., L. femoratus Ab: Karislojo, W. Hellen leg. and Lkor: Saariselkä, B. Poppius leg., L. marmoratus Ka: Vehkalahti, L. Tiensuu leg., L. politus Obb: Kemi, J. Sahlberg leg. and Lkor: Sodankylä, J. Sahlberg leg., L. binotatus Kb: Kitee Juurikka 8.8.1962 V. Makkonen leg., L. picturatus Ok: Suomussalmi, W.Hellen leg., L. sericeus Oba: Hailuoto 11.7.1966 J. Kaisila leg., Obb: Pisavaara naturpark 1.7.1950 N.E. Saris leg., Lkoc: Kittilä 11.8.1967 
M. Meinander leg, and Lkor: Sodankylä Vuotso 9.1954 R. Tynni leg., L. femoralis N: Esbo, B. Poppius leg., L. ignavus Kb: Kontiolahti 24.4.1942 O. Nybom leg., L. pantodapus $\mathrm{Ob}$ : Rovaniemen mlk Pisa 22.6.1951 K. Stenberg leg., L. fuscinervis Ka: Vehkalahti, L. Tiensuu leg., L. dispar N: Ekenäs 665:29 11.6.1977 A. Albrecht leg., L. fenestratus Kb: Kitee Juurikka 29.8.1962 V. Makkonen leg., L. nigriceps Obb: Kemi, J. Sahlberg leg., Lkoc: Muonio, J. Sahlberg leg., Lkor: Pelkosenniemi 29.8.1947 W. Hellen leg., L. fuscicornis Oba: Uleåborg, Coll Nylander, Lkoc: Muonio 25.6.1905 J. Montell leg., and Lkor: Sodankylä, Sundman leg., L. algosus Lkor: Pelkosenniemi Pyhäjärvi 29.6.1951 H. Lindberg leg., L. coenosus Ka: Vehkalahti 23.6.1958 Teirisuo leg., Oba: Oulu, Vuorentaus leg., Obb: Kemijärvi 7.7.1951 J. Carpelan leg., and Lkoc: Muonio 25.7.1920 J. Montell leg., Colpotaulius incisus Obb: Torneå 4.7.1924 H. Lindberg leg. and Lkoc: Muonio, Silfvenius leg., Nemotaulius punctatolineatus Lkoc: Muonio, J. Montell leg., Anabolia laevis Ob: Karunki, J. Sahlberg leg. and Lkoc: Kolari, J. Sahlberg leg., A. concentrica Lkor: Saariselkä, B.Poppius leg., Phacopteryx brevipennis Obb: Pisavaara naturpark 10.7.1950 H. Lindberg leg., Asynarchus contumax Al: ?, Natunen leg., A. thedenii Lkor: Saariselkä, B. Poppius leg., A. lapponicus Lkor: Saariselkä, B. Poppius leg., Arctopora trimaculata N: Borgå Seitlax 30.6.-2.7.1940 A. Nordman leg., Lkoc: Kittilä Pallasjärvi 13.7.1951 J. Kaisila leg., and Lkor: Pelkosenniemi, R. Frey leg., Rhadicoleptus alpestris Obb: Pisavaara nationalpark 18.7.1950 N.E. Saris leg. and Lkoc: Kittilä Pallasjärvi 15.7.1951 J. Kaisila leg., Potamophylax latipennis N: Nurmijärvi 30.8.1.9.1963 M. Koponen leg., St: Karkku 13.8.1967 K. Lahtivirta leg., Sa: Vuoksenniska 4.9.1939 O. Nybom leg., Kb: Tohmajärvi Piilovaara 26.6.1959 V. Mannelin leg., Oba: Uleåborg, Cederhvarf leg., Obb: Rovaniemi 8.1948 J. Carpelan leg., Ks: Kuusamo, J.E. Áro leg., Lkoc: Kittilä, Silfvenius leg., and Le: Enontekiö Hetta, E. Suomalainen leg., $P$. cingulatus Oa: Nurmo 18.8.1951 J. Kaisila leg., Tb: Rautalampi 9.9.1943 Klingstedt leg., Sb: Suonenjoki 13.8.1928 Klingstedt leg., Om: Gamla Karleby 2.8.19?? Hellström leg., Oba: Uleåborg, Coll. Nylander,Lkoc: Muonio, J. Montell leg., and Le Enontekiö, W. Hellen leg., Halesus digitatus Lkor: Sodankylä, Sundman leg., Stenophylax permistus N. Ekenäs lk Tvärminne 22.8.1953 W. Hackman leg., Beraea pullata N: Helsingfors 7.6.1948 W. Hackman leg., Molanna angustata Obb: Pisavaara nationalpark 20.7.1950 N.E.Saris leg. and Lkor: Sodankylä, Sundman leg., M. albicans Oba: Hailuoto, Vuorentaus leg. and Lkoc: Pallastunturi 16.7.1956 Wegelius leg., Molannodes tinctus Obb: Pisavaara naturpark 11.7.1950 N.E. Saris leg., Ceraclea nigronervosa Oba: Hailuoto 9.-12.7.1966 W.Hackman leg., Obb: Rovaniemi 27.6.1929 H. Lindberg leg., Lkoc: Pallastunturi 16.7.1951, Wegelius leg., and Lkor: Sodankylä, Sundman leg., C. alboguttata Om: Ylivieska 9.8.1963 M. Koponen leg., C. annulicornis Oba: Uleåborg, Coll. Nylander, Lkoc: Muonio, J. Montell leg., Lkor: Sodan- kylä, Sundman leg., C. perplexa N: Kerava 15.6.1946 ? leg., C. excisa Ka: Vehkalahti 23.7.1959 L. Fagerström leg., Athripsodes cinereus Oba: Uleåborg, Coll. Nylander, Obb: Kemin mlk Karihaara 28.7.1942 A. Saarinen leg., Lkoc: Muonio Köngäseno 16.7.1956 E. Thuneberg leg., and Lkor: Sodankylä, Sundman leg., A. albifrons Oa: Gamla Karleby, Hellström leg. and Ks: Kuusamo, J. Sahlberg leg., Mystacides niger Obb: Pisavaara naturpark 14.7.1950 N.E.Saris leg., M. azureus Oba: Pudasjärvi 17.8.1965 M. Meinander leg., Obb: Pisavaara naturpark 14.7.1950 N.E.Saris leg., Lkoc: Pallasjärvi 4.7.1953, Wegelius leg., and Lkor: Sodankylä, Sundman leg., M. longicornis Oba: Karlö, H.Frey leg., Obb: Kemin mlk Karihaara 5.8. 1942 A. Saarinen leg., Lkoc: Muonio Köngäseno 16.7.1956 E. Thuneberg leg., and Lkor: Sodankylä Nattanen 13.7.1974 R.Tynni leg., Triaenodes bicolor Oba: Karlö, H.Frey leg. and Obb: Pisavaara nationalpark 20.7.1950 N.E.Saris leg., T. unanimis St: Ahlainen Rankkuu 11.8.1958 V. Lauro leg. and Om: Pyhäsalmi 2.8.1963 M. Koponen leg., Oecetis ochracea Oba: Hailuoto 11.7.1966 J. Kaisila leg. and Obb: Kemin mlk Karihaara 5.8. 1942 A. Saarinen leg., O. lacustris Oba: Uleåborg, Coll. Nylander and Obb: Pisavaara nationalpark 20.7.1950 N.E. Saris leg.

81) Coll. Zool. Mus. Turku University, Turku. Apatania dalecarlica Le: Enontekiö Kilpisjärvi, anon. leg.

82) Coll. Zool. Mus. Oulu University, Oulu. 88 finds. Rhyacophila nubila Obb: Rovaniemen mlk 736:49 15.9.1992 J. Itämies leg., $R$. obliterata Obb: Rovaniemen mlk 736:49 14.9.1992 J. Itämies leg., Agapetus ochripes Kb: Pyhäselkä 692:65 14.7.1983 V.-M. Mukkala leg. and Ks: Kuusamo 7355:608 3.7.1979 O. Siitonen leg., Hydroptila sparsa Ab: Karjaa 26.6.1964 O. Siitonen leg., H. tineoides Oba: Oulujoki 24.7.1963 J. Viramo leg. and Ks: Kuusamo 735:61 18.7.1978 J. Kyrki leg., H. pulchricornis St: Rauma 6794:196 Haurukari 3.8.1971 J. Itämies leg., H. forcipata Ab: Salo 14.6.1964 O. Siitonen leg., Ortotrichia costalis Kl: Simpele 23.6.1947 O. Siitonen leg., Oxyethira flavicornis Ks: Salla 752:61 Värriö 5.6.1986 E. Pulliainen leg., O. frici Ks: Kuusamo 736:60 29.6.1982 O.Siitonen leg., O. mirabilis Ks: Kuusamo 7363:603 6.7.1979 O. Siitonen leg., Wormaldia subnigra Ks: Kuusamo 736:60 Oulanka - 4.9.1977 J. Itämies \& J. Kyrki leg. and Li: Inari 7598:530 29.8.1990 E. Erkinaro leg., Lype reducta St: Pomarkku 30.6.1957 O. Siitonen leg., Neureclipsis bimaculata Oba: Oulujoki 24.7. 1963 J. Viramo leg., Holocentropus picicornis Obb: Rovaniemen mlk. Syväsenvaara 28.6.1991 M. Mutanen leg., H. insignis Oba: Kempele 719:43 9.7.1978 J. Kyrki leg. and Ks: Kuusamo 7355: 608 3.7.1979 O. Siitonen leg., Hydropsyche pellucidula Ab: Pertteli 13.6.1965 O. Siitonen leg., Ceratopsyche silfvenii St: Pomarkku 17.6.1975 O. Siitonen leg., Arctopsyche ladogensis Lkor: Pelkosenniemi 7449:525 21.6.1994 J. Itämies leg., Agrypnia principalis Oba: Muhos 719:45 Metsäntutkimuslaitos 28.8.1977 J. Itämies \& J. Kyrki leg., A. czerskyi Oba: Hailuoto 7218:384 Marjaniemi 26.7.-8.8.1976 J. Itämies \& J. Kyrki leg., Phryganea bipunctata Oba: Oulu 
7219:428 22.6.1988 L. Karstola leg., Oligotricha striata St: Rauma Kortela 10.6.1970 J.Itämies leg., Oligostomis reticulata Oba: Utajärvi 7193:462 Pälli 22.6.1985 E. Lindgren leg. and Le: Enontekiö Kuttanen 256.6.1964 K. Kiianlinna leg., Brachycentrus subnubilus Ab: Lauttakylä 14.6.1957 H. Bruun leg., Lepidostoma hirtum Oba: Oulu 7220:429 30.7.1989 P. Tunkkari \& J. Itämies leg., Apatania wallengreni St: Rauma Mansikkak. 4.6.1970 J. Itämies leg., $A$. auricula K1: Simpele 16.10.1948 O.Siitonen leg., A. zonella Ta: Hausjärvi Hikiä 17.5.1964 J. Viramo leg, and Om: Kannus 709:34 4.-9.9.1978 P. Tokola leg., A. dalecarlica Ks: Salla 752:61 Värriö 21.-22.7.1977 E. Pulliainen leg., Limnephilus rhombicus Oba: Pudasjärvi 724:47 Kipinä 26.6.1993 J. Helkimo leg., L. politus Ks: Kuusamo 76:60 Oulanka -11.9.1977 J. Itämies \& J. Kyrki leg., L. stigma Oba: Oulu 721:42 2.9.1991 P. Tanner leg., L. germanus Al: Finström Husö 11.-12.9. 1975 H. Bruun leg. and Oba: Oulu 7206:428 2.9.1976 J. Itämies leg., L. picturatus Kb: Eno 6952:680 Revonkylä 10.9.1971 A. Pyörnilä leg. and Ks: Salla 751:60 Värriö 9.8.1976 J. Itämies \& J. Kyrki leg., L. externus Ks: Kuusamo 736:60 Oulanka 11.9.1977 J. Itämies \& J. Kyrki leg., L.sericeus Ok: Kajaani 11.7.1970 L. Ekholm leg., L. ignavus Oba: Oulu 720:42 31.8.1978 J. Kyrki leg., L. fenestratus Om: Kannus 709:34 2.-4.9.1978 P. Tokola leg., L.affinis St: Rauma 6792:198 Ruuhiluoto 22.6.1970 J. Itämies leg., L. extricatus Obb: Rovaniemen mlk. 7367:494 Kaihua 20.9.1990 E. Erkinaro leg., L. diphyes Sa: Luumäki 18.7.1970 O.Nybom leg., Om: Kannus 709:34 30.7.-12.8.1978 P.Tokola leg., and Ks: Kuusamo 736:60 Oulanka -4.9.1977 J. Itämies \& J. Kyгki leg., Ironoquia dubia Ab: Halikko 10.9.1972 O. Siitonen leg., Oa: Ilmajoki 697:27, 17.8.1986 V.-M. Mukkala leg.,and Oba: Oulu 720:42 Kiviniemi 3.8.1974 E. Sarkkinen leg., Glyphotaelius pellucidus Oa: Ilmajoki 6972:274 8.9.1984 V.-M. Mukkala leg., Om: Kannus 709:342.-4.9.1978 P. Tokola leg., Oba: Oulu 7207:438 26.8.1977 O. Blomberg leg., and Ks: Kuusamo 7366:603 7.8.1975 J. Kyrki leg., Nemotaulius punctatolineatus Obb: Rovaniemen mlk. 736:49 14.9.1992 J. Itämies leg., Anabolia concentrica Kb: Eno 6952:680 Revonkylä 12.9.1971 A. Pyömilä leg., Asynarchus lapponicus Ks: Salla 751:60 Värriö 18.8.1976 J. Itämies \& J. Kyrki leg., Lenarchus bicornis St:Rauma 6792:200 III. Petäjäs 20.7.1971 J. Itämies leg. and Oba: Kiiminki 722:44 5.-15.7.1972 J. Kyrki leg., Chaetopteryx villosa St: Kankaanpää 3.10.1974 O. Siitonen leg., Ok: Kuhmo 712:66 18.9.1990 J. Viramo leg., and Ob: Rovaniemen mlk. 7367:494 Kaihua 20.9.1990 E. Erkinaro leg., Annitella obscurata Ks:Kuusamo 736:60 Oulanka 11.9.1977 J. Itämies \& J.Kyrki leg., Potamophylax cingulatus Ok: Suomussalmi 726:60 21.7.1979 H. Kärnä leg. and Obb: Rovaniemen mlk. 736:49 14.9.1992 J. Itämies leg., P. rotundipennis Oba: Muhos 719:45 Metsäntutkimuslaitos -22.8.1977 J. Itämies \& J. Kyrki leg., Halesus radiatus Al: Finström Husö H. Bruun leg. and Ks: Kuusamo 736:60 Oulanka -4.9.1977 J. Itämies \& J. Kyrki leg., H.digitatus St: Pomarkku 28.9.1974
O. Siitonen leg. and Ks: Salla 752:61 Värriö 14.15.8.1977 E. Pulliainen leg., H. tesselatus Om: Oulainen 1968 E. Polus leg., Ks: Salla 752:61 Värriö 3.9.1978 E. Pulliainen leg., and Le: Enontekiö 19.8.1951 E.A. Hellman leg., Stenophylax permistus Ab: Houtskär 1.-14.7.1958 H. Bruun leg., Micropterna sequax Kb: Valtimo 7069:587 Nuolijärvi 4.9.1973 J. Itämies leg., Ks: Kuusamo, J. Viramo leg., Hydatophylax infumatus Ks: Kuusamo 7367:608 5.7.1969 O.Siitonen leg., Chilostigma sieboldi Ok: Puolanka 21.4.1967 K. Kiianlinna leg., Brachypsyche sibirica Obb: Rovaniemen mlk. 736:439 Kaihua 4.6.1991 J. Itämies leg. and Ks: Salla 752:61 Värriö 12.9.1989 E. Pulliainen leg., Mystacides niger St: Äetsä 26.7.1967 O. Siitonen leg., Erotesis baltica St: Pomarkku 26.6.1974, O. Siitonen leg., Oecetis furva St: Rauma 6793:200 III. Petäjäs 3.8.1978 J. Itämies leg.

83) Coll. Museum of Natural History in Kuopio, Kuopio. 20 finds. Cyrnus insolutus Kb: Kitee 6877:662 Sarsonlampi 15.6.1988 M. Puustinen leg., Hydropsyche bulgaromanorum $\mathrm{Kb}$ : Kontiolahti 6975:645 Pielisjoki 15.9.1989 H. Hämäläinen leg., Semblis phalaenoides Sb: Haukivuori 6987:505 Sahinjoki 20.7.1986 M. Puustinen leg., Brachycentrus subnubilus Sb: Sonkajärvi 7063:538 Nurmijoki Aittokoski 3.8.1986 M. Puustinen leg., Limnephilus subcentralis Tb: Vesanto 6981:458 Vesijoki 15.6.1979 E. Savolainen leg., Sb: Siilinjärvi 6987:541 Juurusvesi Koivuniemen ranta M. Larikka leg. and Ok: Suomussalmi 7182:604 Jumalisjärvi 2.7.1982 A. Pulkkinen leg., Anabolia nervosa Al: Sund 6702:120 Björbyträsk 5.6.1989 E. Savolainen leg., Tb: Vesanto 6979:463 Iso-Ahvenisesta läht. puro 15.6.1979 E. Savolainen leg., Sb: Siilinjärvi 6988:524 Kehvo Autioranta 11.8.1985 M. Larikka leg., and Kb: Kaavi 6985:577 Kalajoki 13.6.1974 A. Miettinen leg., Chaetopteryx villosa Sb: Heinävesi 6922:584 Luodejoki 21.7.1985 M. Larikka leg., Potamophylax latipennis Sb: Siilinjärvi 6991:542 Rahvonsaari kalliosyvennys 30.6.1985 M. Larikka leg. and Ok: Suomussalmi 723:57 Tervaselkä 15.6.1983 A. Pulkkinen leg., P. cingulatus Kb: Rautavaara 7042:567 Keyritynjoki Siikakoski 18.6.1985 A. Pulkkinen leg., P. nigricornis Sb: Heinävesi 6935:603 Somerjoki 21.7.1985 M. Larikka leg., Halesus radiatus Sb: Nilsiä 7004:547 Kauppisenjoki 16.7. 1985 M. Larikka leg., Silo pallipes Sb: Iisalmi 7055:501 Joutsenjoki 9.8.1987 M. Puustinen leg., Sericostoma personatum Sb: Vehmersalmi 7038:539 Vuorisjoki 3.8.1986 M. Pulkkinen leg., Oecetis testacea Sb: Lapinlahti 7013:523 Pajujärvi 28.8.1987 M. Puustinen leg.

84) Coll. Lounais-Hämeen luonnonsuojeluyhdistys, Forssa. Agraylea cognatella Ta: Tammela 17.7.1970, ? leg.

85) Coll. H. Hämäläinen, Joensuu. 4 finds, all H.H.leg. Rhyacophila fasciata Li: Inari 7629:563 Pikku Taivaljärven laskupuro 30.7.1991 larva, Ithytrichia lamellaris Li: Inari 7640: 556 Juoksemajärven laskupuro 23.9.1991 larvae, Wormaldia subnigra Ab: Pohja 6675:304 Horsmajärven laskupuro 23.7. 1991 larvae, Ceratopsyche nevae Kb: Kontiolahti Pielisjoki 15.9.1989 larvae. 
86) Coll. E. \& L. Laasonen, Helsinki. 32 finds, mostly E.M.L. \& L.L. leg. Rhyacophila nubila Lkor: Inari 759:55 Luttojoki 16.7.1986, Agraylea cognatella Lkoc: Muonio 6.7.1950 O.N. leg. Hydroptila cornuta Li: Inari 760:50 Tolosjoki 2.7.1995, H. tineoides Ka: Kotka, Haapasaari 11.-13.8.1989, Oxyethira flavicornis Obb: Rovaniemen mlk 738:44 Ounasjoki 1.7.1992, O. mirabilis Ka: Virolahti 670:53 Hanski 18.6.1985, Polycentropus irroratus Ks: Kuusamo 729:59 Heinäjoki 24.6.1991, Holocentropus picicornis Ks: Kuusamo 729:60 Kesäjoki 28.6.1995, H. insignis Lkor: Sodankylä Ilmakkiaapa 11.7.1981, Hydropsyche siltalai Ok: Kajaani, linnan rauniot 30.6.1989, Micrasema gelidum Lkor: Sodankylä Vuomaselkä 15.7.1980, Apatania hispida Li: Inari 759:51 Kaunispää 2.7.1996, Limnephilus borealis Obb: Rovaniemen mlk Välijoki 15.8 .1988$, L. picturatus Obb: Rovaniemen mlk 738:44, Ounasjoki Koivusaari 8.9.1990, L. externus Lkor: Sodankylä 754:49 Lohisarrio 30.6.1986, L. femoralis Oba: Oulu 721:42 Nallikari 30.6.1986 and Lkor: Sodankylä 757:50 Männistö 14.7.1991, L. pantodapus Lkoc: Muonio 6.7. 1950 O.N. leg., L. fuscinervis Obb: Hailuoto Marjaniemi 7.7.1965 E. Suomalainen leg., L. elegans Obb: Hailuoto Marjaniemi 6.7.1965 E. Suomalainen leg., L. griseus Li: Inari 760:54 Sarrejänkä 22.-26.7.1995, L.coenosus Lkor: Sodankylä Ilmakkiaapa 11.7.1980, Colpotaulius incisus Obb: Hailuoto Marjaniemi 7.7.1965 T. Suomalainen leg., Nemotaulius punctatolineatus Lkor: Sodankylä 757:54 Sokosti 24.3.( sic!) 1974, Potamophylax latipennis Ta. Kalvola 30.8.1967 O.A. Hellman leg. and Li: Inari 73:58 Näätämö 1.8.1984, P . cingulatus Li: Inari 759:52 Vellinsärpimä 12.7.1986, Goera pilosa Li: Inari 765:55 Kessi 11.7.1995, Molannodes tinctus Lkor: Sodankylä 759:55 Suomujoki 12.7.1985, Athripsodes commutatus Ka: Vehkalahti 28.6.1986 and Obb: Rovaniemen mlk 23.7.1983, Oecetis lacustris Li: Inari 760:53 Hirvaskoski 14.7.1992.

In addition, we take the opportunity to list the caddis fly species found from our summer place Li: Inari 760:54 Kattajärvi from 1970 to 1996: Rhyacophila nubila. Hydroptila cornuta, $H$. tineoides, Oxyethira flavicornis. Polycentropus irroratus. Hydropsyche angustipennis. Agrypnia picta, A. obsoleta, Phryganea bipunctata, Oligotricha lapponica, Hagenella clathrata. $\mathrm{Mi}$ crasema gelidum. Lepidostoma hirtum. Apatania wallengreni, A. stigmatella, A. zonella. Limnephilus rhombicus, $L$. borealis, L. femoratus, L. stigma, L. externus, L. femoralis, L. pantodapus, L. griseus, L. dispar, L. fenestratus, $L$. extricatus, $L$. nigriceps, L. fuscicornis, L. coenosus, Nemotaulius punctatolineatus, Asynarchus contumax, A. lapponicus, Arctopora trimaculata, Rhadicoleptus alpestris, Annitella obscurata, Potamophylax latipennis, $P$. cingulatus, Chilostigma sieboldi. Sericostoma personatum. Molanna angustata, Molannodes tinctus. Ceraclea dissimilis, Atripsodes cinereus, Mystacides azureus, Triaenodes unanimis.

87) Coll. M. \& T. Mutanen, Rovaniemi. Semblis atrata Obb: Tornio 731:38 Kalkkimaa 27.6.1995 T. Mutanen leg.
88) Coll. and archives O. Nybom, Helsinki. 105 finds. Agraylea multipunctata Ks: Kuusamo, J. Kaisila leg., Hydroptila cornuta Ok: Kuhmo, O.N. leg., H. angulata Om: Munsala, J. Waselius leg., H. pulchricornis Sb: Kuopio, E. Räsänen leg. and Oba: Oulu, J. Itämies leg., Ithytrichia lamellaris Sb: Kuopio, E. Räsänen leg., Oxyethira flavicornis Lkoc: Kittilä, E. Räsänen leg., O. tristella Lkoc: Muonio Pallas, O.N. leg., O. simplex Sb: Kuopio, E. Räsänen leg. and Ks: Kuusamo, O.N. leg., O. frici Ok: Kuhmo, M.v. Schantz leg., O. distinctella Ok: Kuhmo, O.N. leg., Stactobiella risi Oba: Oulu, J. Kyrki leg., Tinodes waeneri Ok: Kuhmo, O.N. leg., Psychomyia pusilla Obb: Tervola, O. Siitonen leg., Neureclipsis bimaculata Oba. Oulu, Kangasperkiö leg., Holocentropus picicornis Ok: Kuhmo, O.N. leg., Cyrnus fennicus Sb: Kuopio, E. Räsänen leg., Hydropsyche pellucidula Sb: Warkaus, Malmström leg. and Oba: $\mathrm{Ou}-$ lu, J. Vuorentaus leg., H. saxonica Li: Inari Ivalo, O.N. leg., H. siltalai Oba: Oulu, O. Siitonen leg., Obb: Tervola, O. Siitonen leg., and Lkoc: Muonio, O.N. leg., H. contubernalis N: Helsinki, O.N. leg., Sb: Warkaus, Malmström leg., and Ks: Kuusamo, O.N. leg., Cheumatopsyche lepida Sb: Suonenjoki, Klingstedt leg., Agrypnia pagetana Obb: Tornio, Siltala leg. and Lkoc: Muonio, O.N. leg., A. varia Ok: Kuhmo, O.N. leg., A.czerskyi Sa: Imatra, O.N. leg., Phryganea grandis Oba: Oulu, O. Blomberg leg. and Obb: Tervola, O. Siitonen leg., P. bipunctata Lkoc: Kittilä, O.A. Hellman leg. and Lkor: Pelkosenniemi, O.A. Hellman leg., Oligotricha lapponica Obb: Tervola, O. Siitonen leg., Hagenella clathrata Lkoc: Muonio, A. Aalto leg., Lkor: Pelkosenniemi, A. Aalto leg., and Le: Enontekiö Kilpisjärvi Saarijärvi, A. Aalto leg., Semblis atrata St: Keikyä, Kaario leg., Apatania stigmatella St: Rauma, J. Itämies leg., A. dalecarlica Sa: Rautjärvi, O.N. leg., A. stylata Ta: Valkeakoski, K. Järventausta leg. 12 exx., Limnephilus flavicornis Oba: Muhos, J. Kyrki leg, L L. borealis Oba: Oulu, O. Blomberg leg., L. femoratus Sa: Imatra, O.N. leg. and Kb: Ilomantsi, O.N. leg., L. stigma Lkoc: Kittilä, O.N. leg., L. binotatus Oba: Oulu, Kämä leg., L. germanus Kb: Kitee, Makkonen leg., L. elegans Ok: Kuhmo, O.N. leg. and Obb: Tervola, O. Siitonen leg., L. fenestratus Oba: Oulu, Kärnä leg., L. centralis Oba: Oulu, J. Kyrki leg. and Ks: Kuusamo, O.N. leg., L. sparsus Oba: Oulu, O. Blomberg leg., L. vittatus Le: Enontekiö, Kilpisjärvi, O. Sotavalta leg., L. extricatus Ok: Kuhmo, O.N. leg., Oba: Uleåborg, Nylander leg., Lkoc: Muonio, O.N. leg., and Lkor: Sodankylä, Sundman leg., L. fuscicornis Obb: Tervola, O. Siitonen leg., L. diphyes N: Kirkkonummi, K. Mikkola leg., Kb: Rautavaara, Carpelan leg., and Lkor: Pelkosenniemi, A. Aalto leg., Ironoquia dubia Sb: Riistavesi, E. Räsänen leg., Glyptotaelius pellucidus Lkoc: Kittilä, I. Jalas leg., Nemotaulius punctatolineatus Oba: Oulujoki, ? leg., Anabolia laevis Oba: Oulu, O. Blomberg leg., A. concentrica Ka: Vehkalahti, L. Fagerström leg. and Oba: Kiiminki, J. Kyrki leg., Phacopteryx brevipennis Om: Ylivieska, M. Koponen leg., Oba: Oulu, Kärnä leg., and Lkoc: Muonio, O.N. 
leg., Asynarchus contumax Oba: Kiiminki, J. Kyrki leg., A. thedenii Le: Enontekiö, Hetta, H. Saarenmaa leg., Lenarchus bicornis N: Helsingfors, O.N. leg., Rhadicoleptus alpestris Oba: Haukipudas, J. Kyrki leg. and Lkor: Pelkosenniemi, O.A. Hellman leg., Chaetopteryx sahlbergi, Lkoc: Kittilä, H. Saarenmaa leg., Halesus radiatus Oba: Oulu, J. Kaisila leg., $H$. tesselatus Oba: Kiiminki, J. Kyrki leg., Stenophylax permistus Ta: Kalvola, O.A: Hellman leg., Micropterna sequax Om: Ylivieska, M. Koponen leg., Goera pilosa Sb: Kuopio, E. Räsänen leg., Oba: Oulu, O. Siitonen leg., and Obb: Tervola, O. Siitonen leg., Beraea pullata Lkor: Sodankylä, M.v.Schantz leg., Molanna albicans Obb: Tomio, Siltala leg., M. submarginalis Obb: Rovaniemi, E. Räsänen leg., Molannodes tinctus Lkoc: Muonio, O.N. leg., Ceraclea annulicornis Kb: Pyhäselkä, V. Mannelin leg. and Obb: Rovaniemi, O.N. leg., Athripsodes aterrimus Oba: Hailuoto, E. Suomalainen leg, and Obb: Rovaniemi, O. Siitonen leg., A. commutatus Oba: Uleåborg, Nylander leg., Lkoc: Muonio, O. Siitonen leg., and Lkor: Pelkosenniemi, O. Siitonen leg., Mystacides niger Lkoc: Muonio, O. Siitonen leg., Ylodes reuteri St: Reposaari, V. Lauro leg., Oecetis ochracea Lkoc: Muonio, O.N. leg., O. lacustris Ok: Kuhmo, O.N. leg. and Lkoc: Muonio, O.N. leg., O. notata Sa: Ruokolahti, O.N. leg.

89) Coll. H. Turunen, Tampere. 6 finds, mainly St: Hämeenkyrö, all H.T. leg. Rhyacophila fasciata 6841:310 Äkönmaa Lankoski 9.7.1994, Oligostomis reticulata 6841:310 Äkönmaa Lankoski 10.6.1994, Limnephilus pantodapus St: Ikaalinen 687:30 Seitseminen Kirkkaanlamminneva 12.6.1992, Potamophylax nigricornis 684:30 Pilpuankoski 23.7.1994, Notidobia ciliaris 683:30 Rokkakoskenjoki Sarkkila 27.6. 1994, Molanna submarginalis Ks: Kuusamo 735:59 Käylä Perälampi 15.7.1995.

90) Albrecht, A.: Letter 14.10.1996. 2 finds, both A.A. leg. Apatania zonella N: Sipoo 669:40 Hindsby 29.7.1994, Chaetopteryx villosa Ab: Kiikala 670:31 Kultalähde 4.11.1979.

91) Hirvenoja, M.: Letter 28.10.1996. 28 finds, All Lkor: Sodankylä and M.H. leg. Hydroptila tineoides Korvanen Luiro 22.6.1960, Oxyethira flavicornis Sompiojärvi Kotaoja 6.7.1959, O. simplex Korvanen Tupalehdonoja 22.6.1960, O. frici Sompiojärvi Mutenianjoki 27.6.1959, O. falcata Korvanen Tupalehdonoja 24.6.1960, O. sagittifera Sompiojärvi 23.6.1959, Lype phaeopa Korvanen Posolampi 3.7.1961, Plectrocnemia conspersa Sompiojärvi 24.7.1959, Holocentropus picicornis Korvanen Seitajärvi 8.7.1960, Cyrnus flavidus Sompiojärvi Mutenianjoki 13.6.1959, Agrypnia pagetana Sompiojärvi 8.6.1960, A. obsoleta Sompiojärvi 13.7.1959, A. principalis Sompiojärvi 27.6.1960, Lepidostoma hirtum Korvanen Luiro 24.6.1960, Apatania dalecarlica Sompiojärvi Kotaoja 20.6.1959, Limnephilus subcentralis Sompiojärvi 21.7.1959, L. pantodapus Sompiojärvi Kuusioja 13.6.1959, Glyphotaelius pellucidus Korvanen Tupalehdonoja 17.7.1960, Chaetopteryx villosa
Korvanen Posolampi 30.8.1061, C. sahlbergi Korvanen Tupalehdonoja 7.9.1960, Potamophylax latipennis Korvanen Luiro 12.6.1960, P. cingulatus Sompiojärvi 18.7.1959, Hydatophylax infumatus Korvanen Tupalehdonoja 29.6. 1960, Brachypsyche sibirica Sompiojärvi Kotaoja 25.6.1959, Beraeodes minutus Sompiojärvi Kuusioja 18.6.1959, Molanna angustata Korvanen Seitajärvi 27.6.1960, M. albicans Sompiojärvi Kotaoja 21.8.1959, Oecetis ochracea Sompiojärvi 9.7.1959.

92) Järventausta, K.: Letter 15.10.1996. 19 finds, all K.J. leg. Agrayleya multipunctata Obb: Pello 742:37, 24.7.1995, A. cognatella Lkor: Sodankylä 754:49, 27.7.1996, Oxyethira frici Ab: Kiikala 670:31, 14.6.1995, Holocentropus dubius Obb: Pello 739:36, 23.7.1995, H. insignis Obb: Pello 739:36, 23.7.1995, Limnephilus lunatus Obb: Ylitomio 737:35, 24.8.1995, L. externus Lkoc: Muonio 752:37, 21.8. 1996, L. pantodapus N: Espoo 669:36, 3.7.1993, L. subnitidus Obb: Pello 739:36, 25.8.1995, L.vittatus Obb: Pello 739:36, 23.7.1995, L. nigriceps Le: Enontekiö 768:25, 22.8.1996, Grammotaulius signatipennis Lkoc: Kolari 745:37, 25.8.1995, Anabolia concentrica Ab. Marttila 672:28, 7.9.1995 and Ok: Kajaani 713:55, 10.8. 1994, Asynarchus lapponicus Obb: Ylitornio 737:35, 24.7.1995, Halesus radiatus St: Loimaan kunta 674:27, 2.9.1994, H.tesselatus Ok: Kajaani 713:55, 10.8.1994, Ceraclea senilis Obb: Pello 742:37, 24.7.1995, C. alboguttata Ks.Kuusamo 735:60, 12.8.1994.

93) Saarela, E.: Letters 14.10. and 04.12.1996. 5 finds, mostly E.S.leg. Orthotrichia costalis Ta: Lempäälä 680:32, 9.7.1994, Oxyethira distinctella St. Kullaa 682:24, 8.7.1996, Crunoecia irrorata St: Jämijärvi 686:27, 10.8.1996, 6 exx., Hydatophylax infumatus Kb: Tohmajärvi 690:67, 21.6.1988, Silo pallipes St. Hämeenkyrö 684:31, 4.7.1996 H. Turunen leg.

94) Salokannel, J.: Letters 10.10., 13.11., and 20.11.1996. 13 finds. Holocentropus dubius Kl: Parikkala 683:63 Siikalahti 13.6. 1996 P.R. Sundell \& C. Hublin leg., Agrypnia varia K1: Parikkala 683:63 Siikalahti 4.7.1996 P.R. Sundell \& C. Hublin leg., A. principalis Kl: Parikkala 683:63 Siikalahti 1996 P.R. Sundell \& C.Hublin leg., Limnephilus decipiens Lkoc: Muonio Olos 13.8.1996 H.Turunen leg., L. germanus Kl: Parikkala 683:63 Siikalahti 23.-29.9.1996 P.R. Sundell \& C. Hublin leg., L. lunatus Ks: Salla, kirkonkylän eteläpuolelta H. Turunen leg., L. externus Kl: Parikkala 683:63 Siikalahti 23.-29.9.1996 P.R. Sundell \& C. Hublin leg., L. femoralis Kl: Parikkala 683:63 Siikalahti 25,-31.7.1996 P.R. Sundell \& C. Hublin leg., L. ignavus K1: Parikkala 683:63 Siikalahti 15.-29.9.1996 P.R. Sundell \& C. Hublin leg., Asynarchus contumax Kl: Parikkala 683:63 Siikalahti 4,-10.7.1996 P.R. Sundell \& C. Hublin leg., Halesus digitatus Kl: Parikkala 683:63 Siikalahti 23.29.9.1996, P.R. Sundell \& C. Hublin leg. Sericostoma personatum Kn: Suomussalmi Mustajoki 723:59 K. Mattila leg., Ceraclea excisa Kn: Kajaani Piblajapuro 711: 54, 2.8.1996 T. Piirainen leg. 


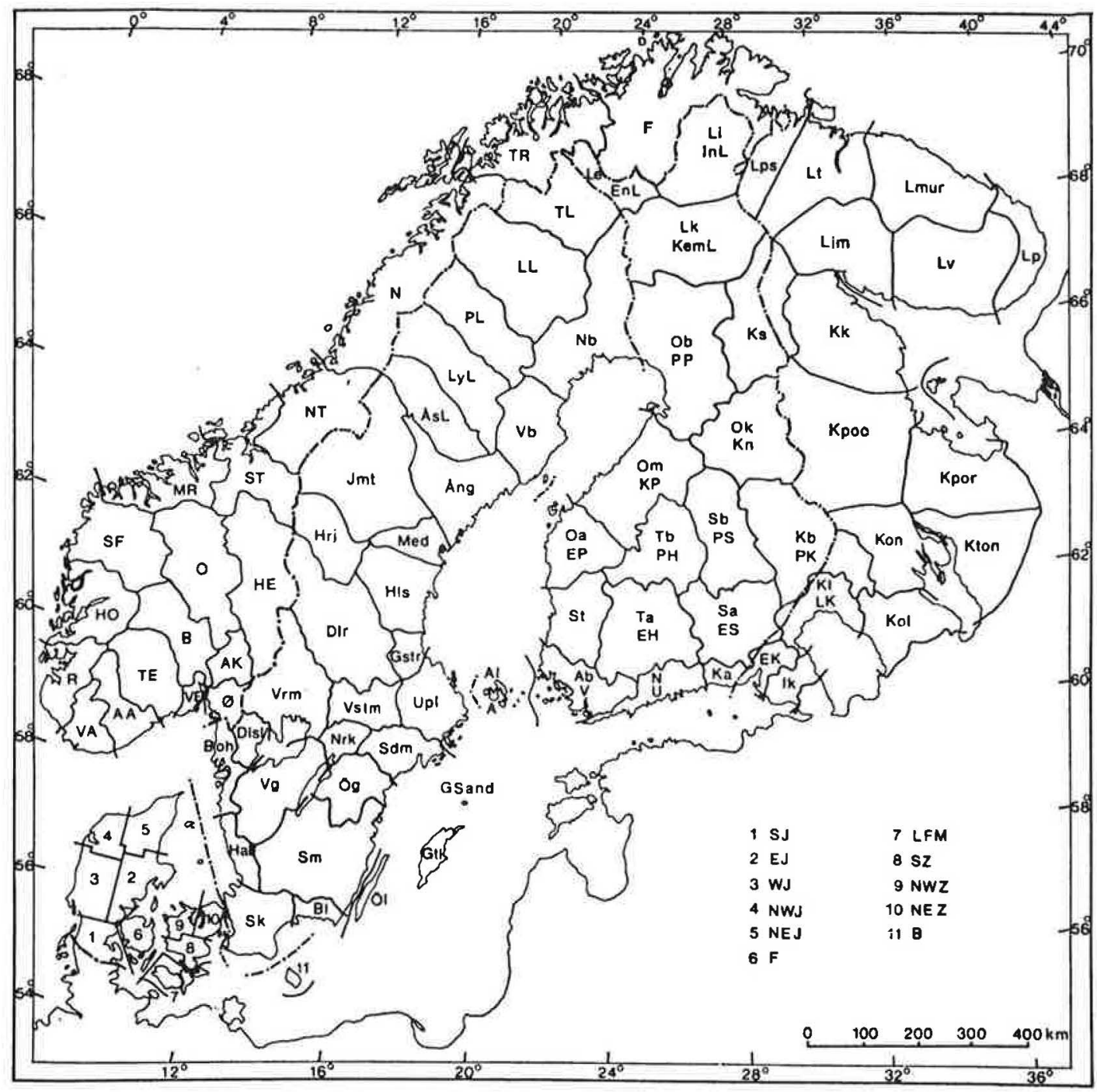

The biogeographical provinces of Fennoscandia and Denmark 